\title{
Towards a new definition of mixed dementia Eniko Kфvari
}

Address: Division of Neuropsychiatry, HUG, Belle-Idıe, Geneva, Switzerland

from International Society on Brain and Behaviour: 3rd International Congress on Brain and Behaviour

Thessaloniki, Greece. 28 November - 2 December 2007

Published: 17 April 2008

Annals of General Psychiatry 2008, 7(Suppl I):S53 doi:I0.II86/I744-859X-7-SI-S53

This abstract is available from: http://www.annals-general-psychiatry.com/content/7/SI/S53

(C) 2008 K $\phi$ vari; licensee BioMed Central Ltd.

Although microscopic cortical infarcts, thalamic and basal ganglia lacunes as well as periventricular and deep white matter demyelination represent the main determinants of cognitive decline in pure vascular cases, their exact role in cases with substantial AD pathology remains unclear. To explore the pathological substrates of mixed dementia, we performed a detailed analysis of lacunar and microvascular pathology in 156 prospectively studied elderly individuals with various degrees of $\mathrm{AD}$ pathology. Cognitive status was assessed prospectively using the Clinical Dementia Rating (CDR) scale; neuropathological evaluation included Braak NFT and A $\beta$-protein deposition staging and bilateral semiquantitative assessment of microvascular ischemic pathology and lacunes; statistical analysis included univariate and multivariate regression models controlling for age. Four independent variables were significantly related to CDR scores. These included Braak NFT staging, A $\beta$ deposition staging, cortical microinfarct as well as thalamic and basal ganglia lacune scores. The concomitant assessment of these neuropathological variables predicted $27 \%$ of the CDR variability. In contrast, white matter lacunes, periventricular and diffuse white matter demyelination as well as focal and diffuse cortical gliosis were not significantly related to CDR scores. In a stepwise approach, the vascular scores explained $15 \%$ of the variability of the presence of dementia, Braak NFT staging $30.4 \%$ and $A \beta$ deposition staging $3.5 \%$. These findings indicate that the clinical expression of the vascular component in mixed cases is highly dependent on lesion type and location as well as severity of concomitant AD-related pathology. In particular, they reveal that the progressive development of CMIs may have a stronger cognitive impact than white matter changes or lacunes visualized by structural neuroimaging in mixed dementia. 